\title{
Innovación en formatos audiovisuales. El uso de herramientas de live streaming en Antena 3 y Univision
}

\author{
MHCJ no 7 | Año 2016 \\ Artículo no 13 (85) \\ Páginas 325 a 349 \\ mhjournal.org
}

\author{
Marina Mancebo García | marinamancebogarcia@gmail.com \\ Universidad Miguel Hernández
}

\begin{abstract}
Palabras clave
Live streaming; Periscope; Facebook Live; nuevos formatos; interactividad

Sumario

1. Introducción 2. Objetivos e hipótesis 3. Marco teórico 3.1 Televisión social, cultura participativa y engagement 3.2 Periodismo móvil y vídeo en directo 3.3 Innovación en formatos de vídeo y narrativas 3.4 Cambio en las rutinas periodísticas 4. Metodología 4.1. Criba y selección de casos 4.2 Análisis de contenido 4.3 Entrevistas en profundidad 5. Resultados 5.1 Análisis comparativo 5.1.1 Número de piezas, temas, duración y alcance 5.1.2 Producción y estrategia 5.1.3 Formatos y lenguajes 5.1.4 Implicaciones profesionales 5.2 La opinión de los expertos sobre live streaming 5.2.1 Producción y estrategia 5.2.2 Narrativas y formatos 6. Conclusiones

7. Recomendaciones 8. Bibliografía
\end{abstract}

\section{Resumen}

El avance de las nuevas tecnologías y la búsqueda de la innovación por parte de los medios de comunicación están favoreciendo un nuevo ecosistema social y mediático. Paulatinamente, los medios audiovisuales apuestan por novedosas técnicas y herramientas para retransmitir vídeo en directo y demandan perfiles profesionales que respondan a las exigencias informativas de la audiencia. El tradicional esquema de emisor-receptor evoluciona hasta lograr una comunicación en la que la audiencia no solo consume información sino que interactúa y participa con el periodista. El consumo de vídeo online en directo crece exponencialmente. Las previsiones indican que para 2018, supondrá el $79 \%$ del tráfico mundial, según Cisco.

Además, el móvil se ha consolidado como el dispositivo por excelencia de consumo, tanto a nivel informativo como social. El objetivo de la presente investigación consiste en conocer cómo están innovando algunos medios audiovisuales con el empleo de herramientas de live streaming. Para ello, el estudio parte de la observación de la producción de dos televisiones que usan vídeo en vivo, Univision y Antena 3 Noticias. Mediante un análisis comparativo, se examina el formato, los contenidos y las estrategias de producción de dos televisiones que usan dos de las herramientas más populares en el mercado: Facebook Live y Periscope. También se emplea una metodología cualitativa con entrevistas a periodistas y expertos en vídeo. Las Jornadas de Periodismo de la Universidad Miguel Hernández y el Congreso ISOJ se han tomado como referencia. Algunas de las conclusiones que arroja la investigación es que el live streaming incorpora diferentes formatos de vídeo, nuevas narrativas nativas de la red, la verticalidad se consolida como tendencia y se requiere un periodista con perfil más audiovisual.

\section{Forma de citar este artículo en las bibliografías}

Marina Mancebo García (2016): “Innovación en formatos audiovisuales. El uso de herramientas de live streaming en Antena 3 y Univision”, en Miguel Hernández Communication Journal, nº, páginas 325 a 349. Universidad Miguel Hernández, UMH (Elche-Alicante). Recuperado el _ de de 20_ de: link del artículo en 


\title{
Innovation in audiovisual formats. The use of live streaming tools in Antena 3 and Univision
}

\author{
MHCJ no 7 | Año 2016 \\ Artículo no 13 (85) \\ Páginas 325 a 349 \\ mhjournal.org
}

\author{
Marina Mancebo García | marinamancebogarcia@gmail.com \\ Universidad Miguel Hernández
}

Keywords

Live streaming; Periscope; Facebook Live; new formats; interactivity

\section{Summary}

1. Introduction 2. Objectives and hypothesis 3. Theoretical Framework 3.1 Social television, participatory culture and engagement 3.2 Mobile journalism and use of live video 3.3 Innovation in video formats and narratives 3.4 Changes in journalist routines 4. Methodology 4.1. Filter and selection of cases 4.2 Analysis of the content 4.3 In-depth interviews 5. Results 5.1 Comparative analysis 5.1.1 Number of pieces, issue, length and significance 5.1.2 Production and strategy 5.1.3 Formats and languages 5.1.4 Professional implications 5.2 Experts opinion about live streaming 5.2.1 Production and strategy 5.2.2 Narratives and formats 6 . Conclusions 7 . Recommendations 8. Bibliography.

\begin{abstract}
The advance of new technologies and the search for innovation in the media are favoring a new social and media ecosystem. Gradually, audiovisual media are posting for novel techniques and tools in order to broadcast live video. This involves a demand professional profiles that respond to the audience informative request. The traditional addresseraddressee outline evolves and reaches a kind of communication where the audience not only consumes information but also interacts and participates with the journalist. Live online video consumption grows exponentially. The predictions indicate that in 2018, it will suppose the $79 \%$ of the world traffic, according to Cisco.

In addition, mobile phone has consolidated as the excellence device of consumption, both informative and socially by adapting the broadcast format to the small mobile screen. The objective of this investigation consists on knowing how some audiovisual media are innovating with the use of live streaming tools. For this reason, the study reports the observation of the two television productions that use live streaming, Univision and Antena 3. By a comparative analysis, it examines the format, contents and the production strategies of two media that use two of the most popular tools in the market: Facebook Live and Periscope.
\end{abstract}

A qualitative method has been used with interviews to journalists and experts. The International Journalism Conference of the Miguel Hernandez University and ISOJ Conference have been taken as a reference. Some of the conclusions that the investigation throws are that live streaming incorporates different video formats and new native narratives. As well, the vertical position is consolidated as a trend and an audiovisual journalist profile is required.

\section{How to cite this paper in bibliographies}

Marina Mancebo García (2016): "Innovación en formatos audiovisuales. El uso de herramientas de live streaming en Antena 3 y Univision”, en Miguel Hernández Communication Journal, $n^{\circ} 7$, páginas 325 a 349. Universidad Miguel Hernández, UMH (Elche-Alicante). Recuperado el _ de __ de 20_ de: Џink del artículo en mhjournal.org] 


\section{Introducción}

En los últimos años han surgido nuevas herramientas que ofrecen la información de modo diferente a los formatos tradicionales. Por eso, parece que asistimos a una época de periodismo distribuido en redes sociales porque el consumo de información actualmente está dominado por las plataformas tanto digitales como móviles. En ese sentido, estas tecnologías condicionan cómo el usuario recibe la información e interactúa con los contenidos.

El auge de los móviles promueve que los contenidos se consuman más en redes sociales. De hecho, los datos confirman que en España, el 53,4\% de los internautas usa las redes sociales para estar informado y que el 93,9\% de las personas que se conectan a internet, lo hacen vía móvil, por delante de otros dispositivos. Lo mismo ocurre en Estados Unidos, donde 39 de 50 sitios de noticias reciben más tráfico desde el móvil que desde el ordenador de mesa. Así, los formatos líquidos son los soportes que se consolidan para el consumo informativo.

El consumo de vídeo online cada vez goza de mayor importancia. Es otra de las tendencias en redes sociales. Los formatos de vídeo reducidos, aquellos que pueden ser consumidos rápidamente desde el Smartphone son los que más éxito están experimentando. Los medios apuestan por esta vía ya que el formato audiovisual arrastra un gran número de seguidores. El $82 \%$ de los internautas españoles opta por el contenido de vídeo online, según datos de comScore. Las previsiones de Cisco también indican que en 2018, este consumo supondrá el 79\% del tráfico mundial.

Varias investigaciones concluyen que incluir vídeo en el contenido produce mayor engagement social y llega mejor al usuario. Además, algunos medios optan por incorporar subtítulos y más contenido meramente visual, porque a pesar de que se visualice más vídeo, el $85 \%$ de lo que se ve, se hace sin escucharlo. De este modo el usuario puede seguir la historia sin perderse nada.

Por otro lado, el live streaming satisface las necesidades de los medios de emitir en directo. Estas herramientas permiten a periodistas y ciudadanos grabar lo que sucede a su alrededor de manera sencilla, ya que basta pulsar un botón para entrar en directo. Facebook y Twitter se han consolidado como dos redes potentes y compiten por la atención del público con el lanzamiento de sus aplicaciones de vídeo en directo online, Facebook Live y Periscope, que ofrecen reproducción vía streaming de acontecimientos en cualquier momento y lugar.

En cuanto a Periscope, fue adquirida por Twitter desde su nacimiento en marzo de 2015, lo que le ha otorgado a la aplicación una significativa ventaja en Estados Unidos sobre su competidor Meerkat. Ahora cuenta con más de 10 millones de usuarios que ven alrededor de 40 años de vídeo al día.

Tras el incipiente éxito de Periscope, Facebook Live, que tenía una función parecida disponible únicamente para cuentas verificadas, se abre para los usuarios de IPhone en Estados Unidos en enero de 2016. Unas semanas más tarde, la posibilidad de retransmitir vídeo en directo se hizo realidad para todos aquellos que estuvieran registrados. Además, esta red social introdujo un cambio en su algoritmo para potenciar el vídeo en directo por encima del vídeo grabado, con una notificación a los seguidores de cada medio, lo que ha impulsado las visualizaciones de streaming. Sin embargo, la evolución no solo reside en el uso de esas herramientas audiovisuales sino en cómo y para qué las emplea el profesional. Algunas de estas innovaciones ya se han dejado ver pero aún quedan muchas funciones por descubrir. El ecosistema de redes sociales se abre hacia un periodismo en primera persona que busca conectar e interactuar con la audiencia de una 
forma más natural y con contenidos que, hasta el momento, no se someten ni a la edición ni a posproducción.

En este contexto, el presente trabajo nace con la siguiente pregunta: ¿Cómo están adaptándose los medios de comunicación para sintonizar con la tendencia global que existe del consumo de vídeo en redes sociales y móviles?

El propósito de esta investigación es estudiar la innovación en formatos audiovisuales, en concreto, de las dos herramientas que han nacido hace un par de años y que están en la cúspide de uso y popularidad.

\section{Objetivos e hipótesis}

El tema de estudio del live streaming tiene aún pocas contribuciones. Ahí reside la importancia y justificación de este fenómeno: aportar más investigación sobre una tendencia en auge: sus usos, importancia y cómo el vídeo en directo podría revolucionar la emisión y el consumo de información.

El objetivo principal es estudiar cómo ha cambiado el modelo de transmisión de noticias con herramientas que reproducen en directo. Esto se llevará a cabo con el análisis del contenido, de las retransmisiones, entrevistas y a partir de ahí se podrá hablar de formatos, implicaciones profesionales y del medio en sí.

En cuanto a los objetivos secundarios, es importante analizar las características que presentan estos nuevos formatos de live streaming con respecto a la producción y elaboración de la noticia, es decir, cómo se prepara el profesional y qué pautas sigue antes de entrar en directo; conocer cómo usa el periodista Periscope y Facebook Live; estudiar a qué tipo de audiencia atraen estas formas innovadoras y, por último, proponer recomendaciones de uso para el profesional en su tarea de transmisión del mensaje.

La presente investigación parte de la hipótesis principal de que el live streaming a través de las herramientas de Periscope y Facebook Live introduce nuevos formatos, lenguajes y narrativas en la tarea periodística informativa, que permiten contar y presentar de modo diferente la información. Por ello, la tarea del profesional cambia para adaptarse a las nuevas exigencias de las redacciones y a la innovación de los medios de comunicación. El periodista abarca más funciones en el mismo tiempo.

Otra de las hipótesis a estudiar es que estas herramientas presentan unos contenidos en los que predomina la naturalidad frente a la rigidez de los de los informativos de televisión. Por consiguiente, aparecen nuevos géneros periodísticos, diferentes a los tradicionales dirigidos a una audiencia presente en redes.

\section{Marco teórico}

Los medios audiovisuales se encuentran en pleno proceso de transformación para adaptarse a las exigencias que imponen tanto las nuevas tecnologías como la audiencia. El presente epígrafe revisa la innovación que están llevando a cabo los medios con el uso del teléfono móvil y el vídeo 
en directo, de qué manera cambian las rutinas periodísticas y cómo captan y conectan con las audiencias.

\subsection{Televisión social, cultura participativa y engagement}

En primer lugar, es necesario conocer algunos términos que están directamente relacionados con el objeto de estudio de esta investigación, el live streaming. La televisión social es un concepto clave que surge por la combinación de redes sociales, segundas pantallas y la televisión (Quintas, González, 2014: 84). Además, el nuevo espectador, con la ayuda de las herramientas que proceden de la Web 2.0, está cambiando la forma tradicional de hacer televisión. "Este aumento de la participación evidencia que los ciudadanos han dejado de ser un mero consumidor de contenidos para participar en la construcción y elaboración de los mismos” (Alonso, 2014).

En esta línea, es necesario remarcar que el usuario se sitúa en el centro del proceso. Unas autoras plantean que el sujeto de la información no es un ser pasivo que se limita a ver la televisión, sino activo porque participa y además genera información (Quintas, González, 2014: 84). Esta situación ha dado lugar al "espectador social" o incluso han nacido nuevos términos como "prosumidor". Con este vocablo se refieren a que aparte de consumir, producen contenidos en la red. Las autoras concluyen que la televisión social ha dado pie al nacimiento de la audiencia social, término que hace referencia a la fragmentación de la audiencia real en función de cómo interactúe en redes sociales.

En el ecosistema digital y en red, los medios de comunicación y en concreto las televisiones, buscan un modelo aún no definido donde la interacción es muy relevante. "La participación de la audiencia ya no es anecdótica en la producción de contenidos televisivos, sino que cada vez se tiene más en cuenta" (Bergillos, 2015: 97).

Para esta tarea, los medios necesitan innovar para conectar con el público y conseguir así la interacción y el interés de sus seguidores. La actitud pasiva de los usuarios ha evolucionado hasta convertirse en una relación de engagement con el medio.

Periscope y Facebook Live se han convertido rápidamente en herramientas para que los periodistas eviten la infraestructura de los medios tradicionales y conecten directamente con la audiencia. "Periscope nace para ser social: no es solo una plataforma de emisión, sino una red social que involucra al emisor y receptor, sin usuarios pasivos" (Ossorio, 2015). En las conexiones, responden a los comentarios del público y muestran sus conocimientos sobre actualidad. En la era de social media, el live streaming les ayuda a forjar su propia marca personal a la vez que hacen frente a las exigencias de la audiencia. Por tanto se requiere que el profesional escriba tuits y esté en directo. No hay excusa para no estar constantemente retransmitiendo (Rugg, Burroughs, 2015: 67).

\subsection{Periodismo móvil y vídeo en directo}

La evolución de la televisión tradicional a un nuevo modelo de transmisión de noticias se ha dejado sentir en el vídeo en directo. La posibilidad de conectar al instante siempre ha sido un 
terreno acotado para las televisiones. Con la aparición de herramientas que emiten en vivo desde un simple teléfono móvil, se amplía el abanico de medios que puedan acceder a esta forma de contar la actualidad.

El vídeo hoy en día lo es todo para los medios por las amplias posibilidades que ofrece. Hace unos meses ya se habló en el Simposio Internacional de Periodismo Online celebrado en Austin del periodismo visual en la era móvil. Uno de los temas abordados en el ISOJ era que lo que triunfa en el formato móvil son resúmenes rápidos, periodismo visual e historias investigadas en profundidad (Zamora, 2015)

Por ello, el periodismo móvil no supone solo un ahorro en los costes sino que permite a los medios contar con imágenes y vídeo de algún hecho noticioso sucedido en cualquier punto del planeta. Además, otro periodista indica que la ubicuidad de las videocámaras permite captar acontecimientos noticiosos, chocantes o inusuales y las televisiones empiezan a buscar y emitir estos vídeos (Díaz Arias, 2009). El tamaño de estas cámara también se posiciona como una ventaja porque consigue que las personas se sientan más cómodas (Frankel, 2016).

Empieza a ser más operativo la grabación con el móvil, tableta o cámara réflex, como del mismo modo se está evolucionando hacia la edición en estos mismo dispositivos (Lavín y Silva, 2015)

Debido al cambio en el consumo de información, los medios tradicionales han tenido que adaptarse a las exigencias que imponen las nuevas tendencias. En su día, las redes sociales supusieron un cambio en el tratamiento informativo como ahora lo están haciendo otras novedosas herramientas de emisión en directo. "Estas aplicaciones van a cambiar la manera en la que las noticias se ven y se producen” (Williams, 2015).

Sin embargo, se requieren más investigaciones en España sobre el uso periodístico de redes sociales y guías de uso para optimizar la plataforma como canal de distribución de contenidos, de marketing y comunicación (Pérez-Soler, 2015). Lo mismo ocurre con los canales de live streaming ya que los medios únicamente han comenzado a experimentar.

\subsection{Innovación en formatos de vídeo y narrativas}

La innovación no solo se encuentra en la participación de la audiencia y en cómo se distribuye el contenido sino que también está patente en el cambio de la narrativa periodística. La aparición de nuevos formatos permite la naturalidad de la comunicación.

La web tiene sus propias narrativas que el periodista José Manuel Noguera analiza en su libro Todos, Todo. Manual de periodismo, participación y tecnología. La narrativa selfie es una de las empleadas en los vídeos que se realizan vía live streaming.

Son individuales por definición, aquellas donde la marca personal del emisor cobra un papel determinante y el mensaje es recibido y consumido para tener el punto de vista del emisor sobre un tema. Aparece, la opinión tanto como la información y sobre todo, bajo formatos audiovisuales (Noguera, 2015: 97) 
En este sentido, el periodista puede realizar un análisis de los hechos, explicar los datos o el contexto, es el valor añadido que aporta como profesional a la audiencia y lo que les enriquece como consumidores de información.

También se ha propiciado la aparición de contenido cuya narrativa se apoya en la combinación e hibridación de lenguajes, medios y plataformas. El foco se ha puesto en la narrativa transmedia: "No es solo una adaptación de un medio a otro. La historia que se cuenta no es la misma, el medio y los lenguajes son diferentes y participan para construir un nuevo mundo narrativo" (Scolari, 2009: 2). Bergillos (2015:86) apunta que el transmedia se apoya en una audiencia activa y participativa que esté dispuesta y sea capaz de explorar el universo narrativo diseñado previamente a través de múltiples plataformas. Por eso cabe remarcar que el nuevo ecosistema informativo, social y conectado necesita contenidos creados por y para internet (Navarro, 2012: $543)$.

Las innovaciones tecnológicas también han facilitado a la producción televisiva posibilidades que propician nuevas experiencias interactivas vinculadas a formatos híbridos (Bergillos, 2015: 96).

Existe una evolución en los géneros y formatos periodísticos más tradicionales, que han pasado a plantearse la forma y el contenido, y de esta manera aprovechar las nuevas posibilidades narrativas que ofrece el entorno digital y adaptarse mejor a los nuevos hábitos de consumo de usuario (Manfredi, et al. 2015: 267)

\subsection{Cambio en las rutinas periodísticas}

El empleo de una tecnología digital innovadora introduce algunos cambios en la tarea del periodista y en el medio, que se vuelve interactivo, personal y omnipresente. Hoy se habla de internet como un espacio en tiempo real, donde se ha incorporado una "nueva lógica del directo que sobrepasa la capacidad de la radio o televisión, con la posibilidad de entrar en directo desde cualquier lugar en cualquier momento" (García Avilés, 2007: 69).

$\mathrm{La}$ actual revolución audiovisual es esencial para los periodistas, que tienen que aprovechar los beneficios del vídeo en directo. Los profesionales hablan de un complemento a las coberturas informativas que realizan en su trabajo día a día. El periodista alemán Ronzheimer recalca que el formato no editado del vídeo en directo es un factor clave para que sus retransmisiones tengan credibilidad e intensidad.

De este modo, la integración del móvil en la rutina del periodista se prefigura como "una extensión natural de las destrezas tecnológicas propias del medio online" (Aguado y Martínez, 2008: 113). No obstante, el periodismo móvil ha provocado cambios tanto en la producción como en los medios de comunicación y periodistas. "Los procesos paralelos de convergencia afectan a estos últimos que, en la actualidad, tienden a acumular labores profesionales que antes eran responsabilidad de especialistas de cada disciplina” (Salaverría y García, 2008: 32).

El perfil del periodista evoluciona para dejar atrás las rutinas tradicionales y adaptarse a las características de un nuevo escenario. La polivalencia es fundamental en las tareas periodísticas ya que el móvil se convierte en una cámara con la que grabar, montar, realizar directos y combinar todas estas funciones de modo correcto es un gran desafío. "El nuevo periodista tendrá que ser 
todoterreno y versátil, manejarse tanto off como online" para así ordenar el caos que genera la sobreinformación y profundizar las historias haciendo uso del potencial de las nuevas tecnologías. (Andueza, Pérez, 2014: 599).

Los departamentos tecnológicos y de innovación de los medios se encuentran ante un gran reto, integrar las aplicaciones que van apareciendo en el trabajo diario del periodista. Algunos expertos realizan comparaciones de las nuevas herramientas con la televisión, incluso llegan a afirmar que es "el futuro de la tv".

\section{Metodología}

Para responder a las hipótesis y objetivos planteados, se empleará la metodología de caso para la realización de un análisis de las retransmisiones, los formatos, qué se hace y cómo funcionan Periscope y Facebook Live, las dos herramientas que se consolidan en dos medios de comunicación. De este modo se podrá establecer una tipología y comprobar cómo y qué se hace en cada una de ellas.

Por este motivo se han realizado entrevistas en profundidad a periodistas, editores y gestores de redes de Antena 3 y de Univision, los dos medios objeto de estudio, para contar con ambas perspectivas sobre el uso y las implicaciones periodísticas. También se incluye el testimonio de cuatro expertos que hablan sobre las herramientas de Periscope y Facebook Live y su importancia dentro del live streaming.

Por último, se ha tomado de referencia el seminario ISOJ y las XI Jornadas Internacionales de Periodismo de la Universidad Miguel Hernández, en la que se realizó un trabajo de campo para analizar el trabajo que un periodista puede realizar con estas redes. Para ello, se retransmitieron con el objetivo de comprobar las indicaciones de los expertos sobre el uso de las aplicaciones móviles objeto de estudio.

\subsection{Criba y selección de la muestra}

Tras la observación de los medios en la industria, se han seleccionado dos medios audiovisuales para realizar el estudio. Por un lado, a Antena 3 por ser pionero en España en realizar vídeos en directo desde cualquier punto del país. Esta televisión apostó por el live streaming para fomentar el empleo de nuevas formas innovadoras de contar lo que ocurre en el mundo y para llegar al público que no les ve por otros canales. Aunque el uso de Periscope empezó como un experimento, se ha convertido en un nuevo medio de información.

Por otro lado, se quería a un medio americano para realizar una comparativa y con la observación de los medios estadounidenses más innovadores, se apostó por Univision ya que son expertos en la plataforma de Facebook Live. Este medio optó por incorporar el uso de Facebook Live desde principios del año 2016 por dos motivos. En primer lugar porque en Facebook ya tienen a su audiencia y por tanto, podían obtener más difusión y alcance inmediato. En segundo lugar porque la propia red social incentiva económicamente a este y otros medios estadounidenses para utilizar la funcionalidad del directo. Desde Univision, creen que es bueno desde el punto de vista 
de ser productores de contenido. Así, lo que ha conseguido Facebook es que los medios promuevan el contenido directamente desde esta red en lugar de desde la página web oficial.

\subsection{Análisis de contenido}

Una manera de determinar la tipología y las características de los vídeos ha sido el análisis de los recursos gráficos lanzados por la cuenta oficial de Antena 3 Noticias en Periscope y Univision en Facebook Live. El periodo de análisis ha tenido una duración de un mes para realizar una clasificación más amplia. La fecha elegida abarca del 26 de marzo al 26 de abril, ambos inclusive. Esta se justifica porque en el mes de marzo se cumplía un año de la puesta en funcionamiento de Periscope. De este modo se puede observar cómo se ha consolidado la herramienta en las redacciones.

Para realizar la comparación con el modelo estadounidense, se ha elegido la misma fecha para los vídeos de Univision y así comprobar si existen similitudes o diferencias en el tipo de conexiones estudiadas.

\subsection{Entrevistas en profundidad}

El trabajo se fundamenta en las declaraciones obtenidas tanto de expertos en estas redes como profesionales que emplean las herramientas. En primer lugar, se enumera a los periodistas que trabajan en los medios de comunicación seleccionados, quiénes han respondido un cuestionario similar pero adaptado a cada aplicación y atendiendo al siguiente esquema de bloques temáticos: preparación, producción y elaboración; formatos, narrativas y tono empleado; pautas, objetivos o estrategias y fortalezas, riesgos o amenazas.

- Gonzalo del Prado, periodista de Antena 3 Noticias de Sociedad y Cultura. Es uno de los redactores de esta cadena más activos en Periscope.

- Mónica Prado, periodista y jefa del Área de Economía de Antena 3 Noticias. Es una de las encargadas de coordinar y decidir las posibles conexiones de live streaming.

- Antonio García Domínguez, ingeniero informático y community manager de Atresmedia. Coordina las redes sociales de la cadena.

- Mariana Marcaletti, editora de redes sociales de Univision. Coordina, fomenta y participa en el uso de la función Live en el medio en el que trabaja.

-Pedro Rojas, periodista y reportero de Univision en Texas. Realiza numerosas coberturas para Univision Noticias. 
A continuación se presenta a cada uno de los expertos.

-Millán Berzosa, doctor en Periodismo y especialista para España y Portugal de Google News Lab. Experto en redes sociales y transformación digital.

-Miquel Pellicer, periodista. Experto e investigador sobre estas herramientas. Ha realizado vídeos para Barcelona Televisió.

-Miguel Ángel Ossorio, periodista y especialista en Comunicación Digital y Social Media.

-Carmela Ríos, periodista y responsable de redes sociales y vídeo social de El Mundo. Experta en nuevas narrativas y usuaria activa en Periscope.

Otra fuente de información han sido los seminarios presenciales o seguidos a través de streaming y redes sociales.

-XVI edición del International Symposium on Online Journalism (ISOJ) que tuvo lugar en Austin los días 15 y 16 de abril de 2016 sobre periodismo digital e ideas innovadoras.

-XI Jornadas Internacionales de Periodismo, dedicadas al Mobile First: comunicación multipantalla, en las que además se realizó un trabajo de campo y experimento con Periscope: la cobertura con esta herramienta de los días 26 y 27 de abril de 2016.

\section{Resultados}

La presente investigación ha examinado la producción, el uso y la implantación de dos de las herramientas más utilizadas en la actualidad, Periscope y Facebook Live, en dos medios de comunicación audiovisuales, Antena 3 y Univision. Este apartado contiene el análisis comparativo entre las dos televisiones estudiadas, atendiendo a diversos criterios temáticos y de contenido además de la opinión de los expertos sobre las características generales que presenta el live streaming.

\subsection{Análisis comparativo}

El análisis de la cobertura live streaming en estos medios se ha dividido en los siguientes subapartados: piezas, temas, duración y alcance; producción y estrategia; formatos y lenguajes e implicaciones profesionales. 


\subsubsection{Número de piezas, temas, duración y alcance}

El primer epígrafe cuantifica el número de piezas que ha emitido cada medio en el período de estudio desde su cuenta oficial. Mientras que Antena 3 solo tiene una única cuenta de Periscope, en la que engloba todas las secciones, Univision cuenta con varias páginas de Facebook divididas por intereses. Así cada emisión se proyecta en su respectiva página, dirigida a un público en concreto. Estas son Univision Noticias, la generalista y en la que más vídeos se realizan; Univision Política; Trends, para un público femenino interesado en estilo de vida, moda o relaciones; Salud, Planeta y Deportes.

En la siguiente figura, se muestra el número total de vídeos que ha realizado cada medio, de modo general, sin atender al contenido.

Tabla 1. Número de piezas emitidas

\begin{tabular}{|c|c|c|}
\hline & Antena 3 Noticias & Univision \\
\hline Número & 25 & 41 \\
\hline
\end{tabular}

Fuente: Elaboración propia

Univision es un medio más activo puesto que en el mismo período, ha utilizado más veces su herramienta como forma de comunicar sobre cualquier tema, a pesar de que su incorporación a la redacción fue unos diez meses más tarde que en Antena 3. El hecho de emitir desde diferentes cuentas, permite la realización de más conexiones.

A continuación, se hará una distinción entre la tipología de los vídeos y los intereses.

Tabla 2. Temática de los vídeos

\begin{tabular}{|l|l|l|l|l|l|l|}
\hline & $\begin{array}{l}\text { Antena 3nivision } \\
\text { Noticias }\end{array}$ & $\begin{array}{l}\text { Univision } \\
\text { Noticias } \\
\text { Trends }\end{array}$ & $\begin{array}{l}\text { Univision } \\
\text { Política }\end{array}$ & $\begin{array}{l}\text { Univisión } \\
\text { Planeta }\end{array}$ & $\begin{array}{l}\text { Univision } \\
\text { Deportes }\end{array}$ \\
\hline Política Acción & 2 & 3 & & 7 & & \\
\hline $\begin{array}{l}\text { Justicia } \\
\text { Ejecutiva }\end{array}$ & 1 & 11 & & & & \\
\hline Medio Ambiente & 1 & 2 & 3 & & & \\
\hline Meteorología & 11 & & & & & \\
\hline Cultura & 3 & 1 & & & & \\
\hline Deportes & 2 & & & & & \\
\hline Economía & 1 & & & & & \\
\hline Casa Real & & & & & & \\
\hline
\end{tabular}




\begin{tabular}{|l|l|l|l|l|l|l|}
\hline Cataluña & 1 & & & & & \\
\hline Tribunales & 1 & & & & & \\
\hline Sociedad & 1 & 2 & 3 & & & \\
\hline Moda & & & 2 & & & \\
\hline Gastronomía & & & 2 & & & \\
\hline
\end{tabular}

Fuente: Elaboración propia

Tabla 3. Localización de los vídeos

\begin{tabular}{|l|l|l|}
\hline & Antena 3 & Univision \\
\hline Plató/Redacción & 2 & 3 \\
\hline Exteriores & & 1 \\
\hline
\end{tabular}

Fuente: Elaboración propia

Como se puede observar, el modelo de Univision presenta notables diferencias en relación al de Antena 3.

Respecto al análisis de temas, el medio ubicado en Miami realiza la mayoría de conexiones de carácter político, normalmente, desde la redacción. En ese lugar conversan periodistas y editores de política sobre los recientes acontecimientos que ocurren. También destaca en este periodo la cobertura que realizaron algunos reporteros sobre la Acción Ejecutiva en la Corte Suprema de Justicia donde se habló sobre la situación migratoria de EEUU.

Otros temas como los relacionados con el planeta, salud o deportes, apenas han tenido importancia en este período y, en general, son las cuentas desde las que menos conexiones se realizan. Por otro lado, cada vez más se proyectan vídeos sobre el tiempo en los que el meteorólogo explica detenidamente el tiempo en el país a través de gráficos y mapas.

Antena 3 otorga menos importancia a las conexiones sobre política pero priman aquellos temas relacionados con la cultura: ruedas de prensa de presentación de series, photocall de actores, música o cine, entre otras así como los que versan sobre fiestas autonómicas. Por último, en deportes destaca el fútbol y utilizan Periscope para mostrar los aledaños del estadio e informar sobre alineaciones.

La redacción es un lugar clave para emitir vídeos. Univision hace un gran uso de este enclave para hablar, sobre todo, de política pero también de economía, realizar entrevistas o recorrer la redacción para comentar las noticias más destacadas de la semana con los periodistas que la integran. Cabe destacar que la mayoría de las conexiones sobre política, se realizan con la redacción de fondo y no desde un lugar exterior. 
Antena 3, como se observa en la tabla, solo ha realizado dos conexiones desde el plató de los informativos. No obstante, este medio se inició por primera vez en Periscope por un tipo de vídeo en el que se mostraba cómo se preparan los presentadores y qué hay entre bambalinas. En el período estudiado, esas dos emisiones desde el plató, presentan una duración mayor que las que suelen realizar en Periscope y arrastran un número mayor de espectadores que cualquier otro vídeo.

Respecto a la duración, no hay un tiempo establecido para cada vídeo. Sin embargo, Univision con Facebook Live suele hacer conexiones de larga duración, excepto las que realizan los corresponsales. Sin embargo, las de Periscope se caracterizan por ser algo más breves ya que la mayoría se realizan fuera de la redacción y se tiene que hacer más uso de las conexiones de datos móviles.

Los datos internos de Univision confirman que para los vídeos con Facebook Live, la duración ideal oscila entre los cinco y diez minutos para llegar así a una audiencia mayor que pueda recordar el contenido de ese vídeo.

El número de usuarios que visualiza el vídeo en directo en cada plataforma también cambia constantemente y es complicado de medir. El título que presenta la conexión es un factor clave de atracción de la audiencia. En líneas generales, Facebook arrastra un número mayor de espectadores de repetición. Esto quiere decir que más personas visualizan el vídeo una vez finalizado. En el caso de Univision, los vídeos pueden tener más de cien mil reproducciones por su perduración en el tiempo.

Sin embargo los de Periscope, hasta el mes de mayo, solo podían ser vistos hasta 24 horas después de su emisión. Ahora, ya se guardan en la cuenta del medio y pueden llegar a miles de personas, pero la cifra, aunque varía en función del tema a tratar, no es superior a los 10 mil espectadores. Facebook, por tanto, tiene un long tail superior y un público potencial mayor. Si bien la página de Univision Noticias en Facebook tiene casi 5 millones de seguidores, la cuenta de Periscope de Antena 3 roza los 75 mil, cuando la de Twitter ronda los 1,5 millones de personas.

En términos de participación, tanto Periscope como Facebook Live generan engagement entre los espectadores. No se puede conocer si el crecimiento de seguidores se debe a la realización de Periscope, pero el community manager de Antena 3, Antonio García Domínguez cree que sí que influye tanto con la información que se aporta como en el componente de innovación. La periodista Mónica Prado añade que "aunque es complicado de medir, sí genera engagement porque ofreces tu marca y cada vez más la gente tiene una relación con la información a través del móvil, aunque luego la televisión es insustituible". En cómputo, todos coinciden en que el uso de estas herramientas genera más participación. 
El Facebook Live, en el caso de Univision, se convierte en la mejor herramienta para asegurar una audiencia para el informativo y que al ser personalizado, implica que el usuario conecte con el periodista como persona.

Esto significa que no se ven los vídeos, en este caso, de la misma manera en un teléfono que en un televisor ni es tan profundo el análisis que se puede realizar en una y otra plataforma. Por tanto, no son excluyentes la televisión y la herramienta de live streaming, sino que el éxito reside en la combinación de elementos.

El periodista Gonzalo del Prado no cree que Periscope se llegue a integrar en el informativo. Las formas de contar las noticias son diferentes. Por eso, pueden ser dos caminos diferentes para hacer información. Y así, el reportero de Univision, Rojas, opina que las conexiones que se realizan pueden servir de gancho para la audiencia que luego pueda ver el reportaje acabado en el informativo y ampliar lo que ha ocurrido en directo.

Con este análisis se observa que el alcance de Facebook es superior y está muy implantado en Estados Unidos. Aunque ambas televisiones son activas en vídeo en directo y hacen frente a las nuevas herramientas para poner al seguidor de redes sociales en el punto donde estén ocurriendo los hechos o ampliar una, la audiencia que tiene Facebook hace más populares las conexiones de Univision.

\subsubsection{Producción y estrategia}

Cada medio y profesional se organiza de una forma diferente dependiendo de la estrategia que sigan. Sin embargo, existe una pauta común: la producción debe ser la misma para realizar una cobertura en directo que para una noticia del informativo. Es necesario contar con los permisos o acreditaciones, documentarse y preparar a la perfección ese trabajo periodístico. A continuación vamos a marcar una diferenciación entre ambos medios.

Antena 3 realiza conexiones tanto desde las delegaciones como desde la sede central. No obstante, la organización se lleva a cabo desde Madrid. Los equipos de informativos, además de la reunión de escaleta que realizan cada día, también realizan otra donde los jefes de cada área deciden qué contenido puede ser interesante para tratar en redes como Periscope o Snapchat.

Aquí tienen cabida aquellos temas que consideran interesantes para la audiencia pero no pueden contar con más tiempo en el minutado del informativo. Una vez decidida qué cobertura se puede hacer, se comunica al periodista o incluso él mismo puede ser activo y proponer conexiones.

Mónica Prado, jefa del área de Economía de Antena 3 Noticias, explica que cuando buscan temas interesantes, no siempre van a la par con lo que puede emitirse en el informativo porque en Periscope tienen cabida todo tipo de temas. Tratan de elegir aquellos contenidos que se adecuen a la forma de contar las cosas que tiene Periscope. Es fundamental identificar lo que puede ser contado en directo.

En Univision recalcan que tienen una programación de los directos para organizarse internamente y ver qué van a lanzar. Dependiendo del tipo de conexión, necesitará más o menos 
preparación. Los vídeos sobre moda o gastronomía son los que más planificación necesitan. La editora de redes sociales, Mariana Marcaletti, explica que en el caso de la moda, sí que preparan un mini guion conversado que no llegan a escribir. En cuanto a los demás intereses, preparan los temas y se apoyan en un papel o soporte móvil para leer los comentarios de la audiencia.

En relación a la organización de las corresponsalías, cada mañana los reporteros realizan una videoconferencia. El periodista Pedro Rojas, encargado del centro de Estados Unidos y Texas relata que primero conocen cómo ha estado el tráfico en digital y redes sociales. Se analiza lo más visto en la web y en Facebook y conforman el menú informativo para ese día. Los productores conversan, desde la sede central en Miami, con los siete responsables nacionales para comentar los temas y las posibles conexiones.

El análisis de los contenidos digitales tiene una gran importancia. En este momento, más del 60\% del tráfico de Univision.com proviene de Facebook, es decir, la audiencia accede a los contenidos a través de esta red social, lo que según Marcaletti excede de su control. Por tanto, dependen de lo que haga esta plataforma en cuanto a sus algoritmos internos. Esto significa que los medios pierden el control sobre lo que producen y sobre el modo de distribuirlo.

Desde Univision ejemplifican la magnitud que tienen los vídeos en directo en Facebook con la recaptura del Chapo Guzmán el 8 de enero. El Facebook Live de ese día tuvo 30.000 reproducciones debido al factor de la notificación, es decir, cuando un usuario sigue a un medio, se le notifica cada vez que está en directo. El número de personas que vieron el vídeo les impresionó, ya que un vídeo normal alcanza miles de vistas, pero no cientos de miles, por lo que Marcaletti concluye que "si Facebook dice que hay que hacer esto, hubiera sido muy tonto por nuestra parte no haberlo hecho".

Como se ha explicado, en ambos modelos, se intenta planificar los siempre y cuando el flujo de la actualidad y la inmediatez lo permitan aunque cada uno sigue una estrategia diferente.

Una de las estrategias de Univision es incorporar más contenido visual. Del análisis que han realizado desde el propio medio de las métricas de consumo, han extraído que la mitad de la gente que ve los vídeos en directo de Univision, no los escucha. Marcaletti lo atribuye a la experiencia de Facebook que hace que se vean mientras se realizan otras tareas. De este modo, se determina la forma de producción y de incluir elementos visuales como gráficas.

Las posibilidades narrativas que presentan los vídeos son innumerables y los medios juegan con la combinación de elementos, desde imagen, sonidos o música hasta infografías o subtítulos para aquellos que ven, pero no escuchan.

La estrategia de producción de Antena 3 engloba a las redes sociales. Los diferentes equipos tienen que trabajar al unísono, con armonía entre las tareas de periodistas, editores y community manager. Cuando se deciden los temas sobre los que se puede hacer un vídeo, se comunica al equipo de redes sociales para que puedan anunciarlo previamente y así llegar a un público mayor.

Por otro lado, se tiene en cuenta en la estrategia el uso de estas herramientas como una forma de marketing para el medio. A pesar de que Periscope no tiene, de momento, ningún sistema de monetización ni aporta beneficios o ganancias directas, sí que lo hace de modo indirecto. Esto quiere decir que utilizar la herramienta influye y beneficia a la marca como cadena. Así lo 
argumenta García Domínguez para Antena 3: "Si vamos haciendo cada vez más cosas, se nota que hay innovación en la cadena. Es marketing pero en un sentido más informativo. Es un extra que ofrecemos a los usuarios por seguirnos".

En el caso contrario, Pedro Rojas afirma que no cabe duda de que Facebook Live sea una herramienta de marketing que sirve para vender contenido y promover otro producto diferente, convirtiéndose en la nueva era de la comunicación.

El objetivo es combinar todas las herramientas que conviven en el ecosistema social en función de los temas y del público, ya que ninguna de ellas está acabada en su totalidad. El papel de los medios es saber exactamente qué tecnología usar en cada momento para hacer cada cosa y llegar a integrarlas en su tarea.

\subsubsection{Formatos y lenguajes}

En este apartado se presenta, desde el punto de vista periodístico, qué formatos, nuevas narrativas, lenguajes y tono presentan en general las herramientas de live streaming.

En el caso de Antena 3 se perciben formatos según el criterio temático (de qué trata la noticia sobre la que se realiza la cobertura), el tratamiento (informativo, lúdico o interpretativo), la estructura y el formato de emisión (horizontal o vertical). Son los siguientes:

Según el tema de la conexión, los vídeos se emiten porque los periodistas van a cubrir las diferentes noticias que ocurren. Por ello, pueden retransmitir un estreno de cine, una fiesta autonómica, la Semana Santa o un incendio que se haya producido. Los temas son tan variados como las noticias que puedan transcurrir en un día.

En cuanto al tratamiento, las noticias se dividen por el criterio informativo, que es estrictamente de actualidad, como se pudo observar en la cobertura de los atentados de París; conexiones lúdicas, aquellas menos urgentes de nivel informativo pero sí interesantes para la audiencia como la presentación de la serie La Embajada (Carlos Sedes, Eduardo Chapero-Jackson, 2016) y el formato interpretativo que incluye el contexto y la opinión, donde la figura del experto tiene máxima importancia.

Los vídeos de este medio suelen tener la misma estructura: el periodista comienza la retransmisión con la cámara interna, se presenta y seguidamente cambia de plano para explicar el tema en cuestión. El núcleo central del streaming puede contar con entrevistas o simplemente con el desarrollo de los acontecimientos. Para la despedida, se suele recurrir de nuevo a la imagen del periodista.

Atendiendo al formato de emisión, Periscope está diseñado para cambiar la mentalidad de vídeo horizontal y probar otra forma nueva de grabar, adaptada al dispositivo y pantalla móvil. Aunque no todos lo utilizan, ya que depende del periodista que en ese momento vaya a realizar la conexión, 14 de 25 vídeos lanzados en Antena 3 en el período estudiado se retransmitieron en vertical, 10 en horizontal y uno combinó ambas formas. Esto demuestra que los medios van 
abandonando progresivamente la mentalidad de emitir en horizontal para dejar paso a otras alternativas, una de las predicciones que anunciaba el Reuters Institute News Report para 2016.

En Univision el formato de emisión es diferente, ya que los vídeos suelen ser cuadrados por defecto siempre y cuando se utilice el móvil como dispositivo de grabación. Cabe remarcar que aunque empleaban al inicio el móvil para las conexiones, algunas de las que se están realizando en la actualidad son a través de unas cámaras especiales que conectadas al teléfono móvil, permiten editar en tiempo real y obtener una mejor calidad de imagen. Esta nueva tecnología, incorporada a los vídeos emitidos desde junio, ya supone una evolución a la naturalidad de los formatos actuales porque además de estar en directo, se añaden elementos como el logo de la cadena o rótulos.

En cuanto a los formatos que se están utilizando en los vídeos de Univision, se encuentran los siguientes:

Vox pop. Univision Trends emitió un vídeo por el Día Internacional del Beso en una cafetería donde entrevistaron a gente para que contara sus historias y experiencias. Se trata de un formato híbrido que combina entrevistas en sitios de ocio o culturales.

Entrevista. Interesante cuando se cuenta con un experto. A Univision acudió un contador para responder preguntas y solventar dudas acerca de la declaración de impuestos.

Visual. No contiene texto, se deja que las imágenes o los hechos hablen por sí solos. Dentro de la cobertura de los acontecimientos de la Corte Suprema, se retransmitió el discurso de los inmigrantes reunidos enfrente de este edificio y el periodista no interviene.

Entretenimiento. Mesa redonda al inicio de temporada de Juego de Tronos.

Recitales en vivo de música. La visita a Univision de la Florida Gran Opera. Se escucha a la soprano, protagonista de la ópera.

Eventos noticiosos en vivo. Uno de los formatos más utilizados en redacciones por el fin periodístico que conlleva. Por ejemplo, las inundaciones que tuvieron lugar en Houston.

La narrativa utilizada en los Facebook Live de Univision es menos secuencial y ordenada que el streaming tradicional, por tanto se aleja de la pirámide invertida típica del periodismo. No comienzan con lo más importante porque muchos usuarios se unen al vídeo más tarde y de este modo se perderían el inicio y la información más relevante. Sin perder de vista la estructura de principio, medio y fin, Mariana Marcaletti propone un tratamiento de espiral, parecida a los recursos narrativos de la radio, circular, repetitivo y reiterado. Al contrario que en la televisión tradicional, no está de más redundar y hacer pequeños recordatorios.

El modo de uso de Periscope por Antena 3, condiciona su narrativa ya que su base son las noticias. Por ello se trata de conexiones más cortas que no inciden tanto en redundar, sino en explicar los acontecimientos y responder a los comentarios, dudas o preguntas que le surja a la audiencia.

En cuanto al lenguaje, todos los periodistas entrevistados coinciden en que es más fresco. El tono utilizado presenta notables cambios, es cercano y rompe con las barreras que existían en 
televisión donde el mensaje era unidireccional. De este modo, el poder interactuar con la audiencia hace que el tratamiento sea de tú, algo más informal, en lugar de hablarles de usted, propio de las televisiones.

Mientras que la televisión se basa en un guion, una gran preparación y la formalidad que conlleva, lo que implica más rigidez en las palabras empleadas, Facebook Live o Periscope son medios a través de los cuales se puede hablar de forma improvisada y espontánea.

La naturalidad de esta forma de contar, unido al lenguaje y tono empleado, se convierte en un añadido extra para la audiencia, donde el filtro es la propia realidad. Se ofrece al público algo más de lo que podrán ver en un informativo o de lo que el medio pide al periodista. Con ese contenido extra quieren acercarse a los espectadores, como un modo de agradecerles que estén ahí y les sigan cada día.

\subsubsection{Implicaciones profesionales}

El periodista se enfrenta a nuevos retos y desafíos con la implantación de las herramientas de live streaming en los medios de comunicación. Además de las tareas básicas derivadas de su actividad, se le añade otra más: el uso de las redes sociales. En la actualidad, se suma la realización de vídeo en vivo para contarle a la audiencia qué sucede en un determinado momento y así informarle antes de que enciendan el televisor para ver las noticias.

Una de las recientes novedades es que el periodista puede dar acceso al público a contenidos que nunca antes había visto y que no tiene la oportunidad de seguir por redes sociales ni por televisión. Un ejemplo fue la presentación de la serie Vis a Vis (Jesús Colmenar, 2015). Gonzalo del Prado retransmitió esta rueda de prensa a la que hasta ahora, solo tenían acceso los medios de comunicación. Así, los seguidores de la cuenta de Antena 3 Noticias pudieron ver cómo se realizaba, cómo hablaban productores y guionistas de la serie e incluso preguntar sobre la nueva temporada.

Este tipo de conexión se considera un extra que se da a la audiencia. Se muestra el trabajo que realiza cada día el profesional desde dentro, y gracias a su conocimiento sobre el tema, presenta a los protagonistas que aparecen en el vídeo, les entrevista y responde en directo a las cuestiones que plantean los espectadores, lo que le otorga frescura a la emisión. Además, ese directo "tiene personalidad única porque representa un lugar, un punto geográfico, una circunstancia social o círculo cultural", asegura Rojas.

Para realizar estas tareas, se requieren ciertas cualidades del profesional. En primer lugar, el periodista debe tener en cuenta que no habla desde su cuenta personal, sino desde la del medio en cuestión. Eso implica tener una preparación mayor y ser muy riguroso con el contenido. Representan a un medio y no pueden entrar en los comentarios negativos que se generen. Por otro lado, si el periodista se interesa por las nuevas herramientas y las prueba, podrá decidir si resultan útiles o si aportan interés a la historia. No puede resistirse a su uso ya que si el público está, el profesional también porque es una forma de fidelizar a los usuarios.

Pedro Rojas sugiere que es mejor hacer uso de las herramientas por gusto y no por obligación. De este modo, aunque es añadir un extra al trabajo diario, se puede desarrollar una relación más íntima y cercana con el público que ve los vídeos y así el periodista, haciendo uso de sus 
conocimientos, puede ofrecer a la audiencia otra perspectiva de lo que ocurre de manera inmediata a modo de adelanto informativo.

Las necesidades de internet y las novedades en innovación en materia de live streaming, requieren profesionales con un perfil orientado a la narración visual, capaz de dominar los encuadres y con nociones sobre vídeo. La creatividad es otras de las cualidades que proponen desde estos medios.

\subsection{La opinión de los expertos sobre live streaming}

El análisis de los modelos de Antena 3 y Univision se ha complementado con entrevistas a expertos para conocer las características del live streaming y lo que aportan el uso de las aplicaciones de vídeo en vivo a los medios y periodistas.

\subsubsection{Producción y estrategia}

Los expertos proponen una serie de pautas que tienen que seguirse antes de realizar una retransmisión. El directo implica que el periodista tenga algo noticioso de contar, con cierta relevancia y que tenga a su disposición los medios técnicos necesarios, es decir, asegurarse de disponer conexión a internet y batería en el teléfono móvil. Además, es necesario preparar ese trabajo periodístico en Periscope o Facebook Live como si fuera para cualquier otro medio.

El periodista Miquel Pellicer, realiza una distinción desde el punto de vista en el que se va producir el evento: si es un breaking news, está ocurriendo en el momento, por lo que la planificación la otorga la experiencia. En cambio, si es un evento programado, se puede guionizar y pensar cómo presentarlo, además de poder contar con expertos que realicen un análisis sobre el tema.

En cuanto al contenido, cabe destacar a Millán Berzosa quién explica que lo que funciona no es adaptar el contenido de un medio a otro sino generarlo desde el propio, en este caso, crear algo específico desde Periscope o Facebook Live. De este modo se crea una experiencia diferente para el usuario y le hace comprender y analizar situaciones desde un formato visual e interactivo.

Respecto a la audiencia, los medios, además de decidir qué cobertura desean realizar, también tienen que tener en cuenta a qué público se dirigen. Eso implica elegir entre Periscope y Facebook Live, dependiendo de dónde alcancen más usuarios puesto que Facebook se dirige a una comunidad más amplia y los medios consiguen una audiencia potencial superior.

Los usuarios son imprescindibles, puesto que se sienten parte de este proceso. Por ello, la estrategia debe mirar por y para ellos. La interacción permite preguntar, solventar dudas y descubrir historias. Cabe mencionar al periodista Ronzheimer, quién ha realizado un viaje acompañando a un grupo de refugiados para saber más acerca de ellos. En sus conexiones de Periscope, la audiencia preguntaba por qué llevaban gafas de marca o poseían un Smartphone. El conductor de esa retransmisión hacía de intermediario del público y preguntaba directamente cuestiones que los mismos refugiados explicaban. De este modo se observa una tendencia ligada al live streaming: el periodismo inmersivo. 
Carmela Ríos sostiene que el ecosistema móvil se basa en un diálogo multimedia que deja atrás la función emisor-receptor y sobresale la cultura de la imagen y la generación de contenido para enriquecer nuevos modelos informativos. En una era en la que los medios se enzarzan en una lucha por captar la atención de la audiencia, es necesario implicarla con contenidos atractivos y apelar así, al servicio público de periodismo.

El vídeo cada vez lo consumen más personas y por este motivo los medios cuidan su estrategia audiovisual. Ríos considera que aunque aún no han realizado un desarrollo comercial o publicitario de Periscope y Facebook Live, llegará de un momento a otro. Ahora es el momento de la tecnología.

Como se ha apuntado, aunque otras herramientas llegaron antes, no lo hicieron en el tiempo adecuado. Cada aplicación presenta unas particularidades concretas pero lo que marca la diferencia es la disponibilidad de servicio y la usabilidad. Algunos expertos consideran que Periscope es una herramienta de marketing puro ya que tiene detrás a un gigante como Twitter, por lo que aprovecha su base de usuarios para crecer. En cuanto a Facebook, Pellicer señala que esta plataforma ha hecho que algunos medios estén promoviendo contenidos directamente en Facebook Live, por lo que poco a poco están abandonando la permanencia en su sitio web.

\subsubsection{Narrativas y formatos}

En este epígrafe, se propondrán otros formatos que se pueden encontrar en los vídeos en directo, atendiendo a tres criterios:

Según el lugar desde el que se realice la conexión, Pellicer diferencia entre los formatos parecidos a televisión, desde plató y las conexiones en directo a modo de stand up. Después se encuentran los formatos híbridos como realizar entrevistas en lugares culturales o de ocio.

En relación al contenido, hay dos vertientes. La oportunidad del directo cuando ocurre un acontecimiento importante, el espíritu CNN+ "está pasando, lo estás viendo" o el contexto y la opinión, donde hablan los expertos, según la propuesta de Ríos.

Desde el punto de vista de la emisión, Millán Berzosa distingue entre el streaming puro, de flujo continuo, como Youtube, o discontinuo, como los micro vídeos de Snapchat o Periscope, que se proyectan varios sobre el mismo tema. El formato de grabación es vertical u horizontal y la perduración del vídeo, ahora ilimitada tanto en ambas herramientas.

Atendiendo a la narrativa utilizada, puede ser pura, cuando el periodista no interviene en los acontecimientos y las imágenes aportan toda la información o, por otro lado la cobertura similar a las conexiones en directo de las televisiones, donde el periodista es conductor de la historia.

La periodista Ríos señala que lo importante es rellenar con narrativas periodísticas cada herramienta y tecnología. Además, añade que el futuro es la gimnasia narrativa, es decir, encontrar la mejor forma de contar la noticia. Para ello, hay que saber elegir la aplicación adecuad. Pellicer mantiene que las herramientas se pueden complementar, para beneficiarse de las ventajas y características de cada una. Otros como Ossorio opinan que aunque las dos plataformas emiten vídeo en directo, Facebook tiene una base de seguidores más involucrados.

Las redes sociales han acabado generando contenidos periodísticos, un fenómeno que Carmela Ríos ha definido como géneros periodísticos móviles. Es más, uno de los contenidos que se crean 
para redes es el live streaming. Esto se debe a la multitud de posibilidades que ofrecen. Durante las Jornadas Internacionales de Periodismo, se remarcó la importancia de integrar la cobertura móvil en la tradicional de los medios, para hacer frente a las necesidades que les surgen a los usuarios.

\section{Conclusiones}

Tras la exposición de los resultados, se extraen las siguientes conclusiones sobre el uso de las herramientas de vídeo en directo de acuerdo a las hipótesis planteadas:

El live streaming incorpora nuevos formatos de vídeo que se pueden combinar en función de la finalidad de la conexión, ya que hay múltiples posibilidades y no son excluyentes entre sí. Los medios tradicionales realizan experimentos a través de estas herramientas que no hacen en otros vídeos.

La verticalidad es una tendencia en las conexiones de Periscope porque se adapta al dispositivo móvil. Cada vez más periodistas optan por este formato en lugar de la horizontalidad, por lo que la mentalidad en cuanto a la forma que presentar el vídeo evoluciona.

Las narrativas utilizadas son nativas de la red, con una estructura circular y repetitiva. No se empieza con lo más importante porque se unen usuarios al vídeo progresivamente. El periodista decide si interviene o no en la narración. El lenguaje es más fresco, cercano, improvisado y desenfadado. Se permite el uso de coletillas o frases hechas propias del habla cotidiana.

La cultura del móvil y las redes sociales han desarrollado nuevos géneros periodísticos móviles, diferentes a los tradicionales. Uno de los contenidos específicos que presentan es la emisión en directo o live streaming.

La audiencia que atraen estas formas innovadoras es joven, nativa de redes y aquella que no puede consumir información por otras vías. Los medios eligen en que aplicación lanzar su contenido dependiendo del tipo de público al que quieren llegar. Lo que funciona es crear algo único desde la propia herramienta y no las adaptaciones de contenidos de un medio a otro para que el usuario comprenda y analice la información de forma visual.

Se rompe el modelo de emisión tradicional de uno a muchos. Se desdibujan las figuras de emisorreceptor porque hay feedback con la audiencia y el usuario es consumidor y productor de contenido. No es solo un vídeo, sino que se proyecta periodismo interactivo en vivo, ya que se crea una experiencia para el usuario que se siente parte activa del proceso comunicativo.

La tarea del periodista sufre transformaciones. Tiene que ser curioso, implicarse y tener ganas de probar herramientas para contar las historias de modo diferente, según las exigencias de la tecnología. Presenta un perfil más audiovisual, es todoterreno, sabe escuchar y está muy preparado ya que las aplicaciones le permiten más tiempo para explicar lo que sabe. 
La popularidad y la consolidación de Facebook Live y Periscope se debe a estar ligada a dos potentes redes sociales como son Facebook y Twitter, ya que el contenido se emite directamente desde la primera plataforma y está enlazado en el timeline de la segunda. Además, se accede fácilmente a ellas.

Los medios de comunicación han perdido el control sobre lo que producen y sobre cómo lo distribuyen. Es el caso de aquellos que usan Facebook porque esta red, a modo de gatekeeper, es quién marca qué contenidos desea primar por encima de otros y quién está pagando a algunos medios americanos para que usen sus funciones y lancen contenido o noticias directamente en la red social. Por tanto, los medios ponen más contenido nativo en Facebook y menos en su página web.

\section{Bibliografía}

Aguado, J. M. y Martínez, I. (2008): "La comunicación móvil en el ecosistema informativo: de las alertas SMS al mobile 2.0". Trípodos, n ${ }^{\circ}$ 23, España, pp. 107-118. Recuperado de http://goo.gl/bsTozn (Consultado el 13 de abril de 2016)

Alonso González, M. (2014): "Audiencia Social: el telespectador comienza a participar en los contenidos televisivos". Ámbitos: Revista internacional de comunicación, $\mathrm{n}^{\circ}$ 25, España, junio, pp.77. Recuperado de http://goo.gl/7E3OU7 (Consultado el 31_de marzo de 2016)

Andueza López, M.B y Pérez Arozamena, Rosa (2014): “El móvil como herramienta para el perfil del nuevo periodista”. Historia y Comunicación Social Vol. 19, $\mathrm{n}^{\circ}$ Esp, España, enero, pp. 591602. Recuperado de http://goo.gl/CSFw39 (Consultado el 31 de marzo de 2016)

Bergillos, Ignacio (2015): Participación de la audiencia y televisión en la era digital. Propuesta de análisis y evolución de las invitaciones a la participación en la tdt y en otras plataformas (Tesis de Doctorado). Universidad Autónoma de Barcelona, España. Recuperado de http://hdl.handle.net/10803/308326

Díaz Arias, Rafael (2009): "El video en el ciberespacio: usos y lenguaje”. Comunicar: Revista científica iberoamericana de comunicación y educación, n⿳33 v.17, España, octubre, pp. 63-71. Recuperado de https://goo.gl/yNesMd (Consultado el 31 de marzo de 2016)

Dredge, S. (2015). “How live video on Periscope helped 'get inside' the Syrian refugees story”, en The Guardian, Reino Unido, septiembre: https://goo.gl/5Z80Cg Fecha de consulta: 19 de febrero de 2016 
García Avilés, J.A (2007): "Nuevas tecnologías en el periodismo audiovisual”. Revista de la Facultad de Ciencias Sociales y Jurídicas de Elche, Vol.1, $\mathrm{n}^{\circ}$ 2, España, marzo, pp. 59-75. Recuperado de https://goo.gl/wHsZnH (Consultado el 31 de marzo de 2016)

Manfredi Sánchez, J. L., Rojas Torrijos, J. L., Herranz de la Casa, J.M. (2015): “Innovación en el periodismo emprendedor deportivo. Modelo de negocio y narrativas". El profesional de la información, Vol. 24, $\mathrm{n}^{\circ}$ 3, España, mayo-junio, pp. 265-273. Recuperado de http://goo.gl/gMf4oH (Consultado el 23 de marzo de 2016)

Navarro, F. (2012): Análisis prospectivo sobre la evolución de las herramientas de participación social en cibermedios españoles de diversa matriz mediática (Tesis de Doctorado). Universidad Miguel Hernández de Elche, España.

Noguera Vivo, J.M (2015): Todos, Todo. Manual de periodismo, participación y tecnología. Barcelona: UOC.

Ossorio Vega, M.A (2015): "Periscope, el vídeo en directo para todos" en Internet Media Lab, España, junio. Recuperado de http://goo.gl/FsM3aL (Consultado el 31 de marzo de 2016)

Quintas Froufe F, N. \& González Neira, A. (2014): "Audiencias activas: participación de la audiencia social en la televisión”. Comunicar, no 43, España, julio, pp. 83-90. Recuperado de http://goo.gl/h3M8Um (Consultado el 31 de marzo de 2016)

Reuters Institute (2016): Journalism, media and technology predictions 2016. Recuperado de http://goo.gl/5z04uf (Consultado el 11 de abril de 2016)

Rugg, A. y Burroughs, B. (2016): "Periscope, live-streaming and mobile video culture". Institute of Network Culture. Amsterdam, pp. 64-72. Recuperado de http://goo.gl/icDy20 (Consultado el 16 de abril de 2016)

Salaverría, R., García Avilés, J.A (2008): "La convergencia tecnológica en los medios de comunicación: retos para el periodismo". Trípodos, n²3, España, pp. 31-47. Recuperado de http://goo.gl/Oj16QD (Consultado el 13 de abril de 2016)

Scolari, C.A. (2009): "Transmedia Storytelling: Implicit Consumers, Narrative Worlds, and Branding in Cotemporary Media Production". International Journal of

Communication, n³, España, enero, pp. 586 - 606. Recuperado de http://goo.gl/niavMA (Consultado el 16 de abril de 2016) 
Williams, O. (2015): "Periscope and live video are changing the internet forever" en The Next Web, Amsterdam, marzo. Recuperado de https://goo.gl/Fx90Lx (Consultado el 31 de marzo de 2016) 


\section{(c) (i) (2)}

Licencia Creative Commons

Miguel Hernández Communication Journal

mhcj.es

Forma de citar este artículo en las bibliografías

Marina Mancebo García (2016): "Innovación en formatos audiovisuales. El uso de herramientas de live streaming en Antena 3 y Univision”, en Miguel Hernández Communication Journal, nº7, páginas 325 a 349. Universidad Miguel Hernández, UMH (Elche-Alicante). Recuperado el — de de 20 _ de: [link del artículo en mhjournal.org] 